\title{
Overexpression of TCP8 delays Arabidopsis flowering through a FLOWERING LOCUS C- dependent pathway
}

\author{
Xiaoyan Wang ${ }^{1 * \dagger} \mathbb{B}$, Xintong $\mathrm{Xu}^{1+}$, Xiaowei Mo ${ }^{1}$, Luyao Zhong ${ }^{1}$, Jiancong Zhang ${ }^{1}$, Beixin $\mathrm{Mo}^{1}$ and Benke Kuai ${ }^{2^{*}}$
}

\begin{abstract}
Background: Flowering is a key process in the life cycle of plants. The transition from vegetative to reproductive growth is thus under sophisticated regulation by endogenous and environmental signals. The plant-specific Teosinte Branched 1/Cycloidea/Proliferating Cell Factors (TCP) family transcription factors are involved in many biological processes, but their roles in regulating flowering have not been totally elucidated.

Results: We explored the role of Arabidopsis TCP8 in plant development and, especially, in flowering control. Overexpression of TCP8 significantly delayed flowering under both long-day and short-day conditions and dominant repression by TCP8 led to various growth defects. The upregulation of TCP8 led to more accumulated mRNA level of FLOWERING LOCUS C (FLC), a central floral repressor of Arabidopsis. TCP8 functions in an FLCdependent manner, as TCP8 overexpression in the flc-6 loss-of-function mutant failed to delay flowering. The vernalization treatment could reverse the late flowering phenotype caused by TCP8 overexpression.
\end{abstract}

Conclusions: Our results provide evidence for a role of TCP8 in flowering control and add to our knowledge of the molecular basis of TCP8 function.

Keywords: TCP8, Flowering, FLC, Vernalization, Arabidopsis

\section{Background}

The transition from vegetative to reproductive growth is one of the most important processes in the life cycle of flowering plants. As a result, flowering is under strict and sophisticated regulation by multiple endogenous cues and environmental signals [1]. Many studies using the model plant Arabidopsis thaliana (A. thaliana) have identified major flowering-related genes, these genes were found to be involved in photoperiod, vernalization, gibberellin, aging, temperature, and the autonomous pathways [2-4]. For example, the central floral repressor FLOWERING LOCUS C $(F L C)$ is involved in both the vernalization and the autonomous pathways. During vernalization, FRIGIDA (FRI) activates $F L C$ expression while prolonged cold repressed $F L C$ expression $[5,6]$. In the autonomous pathways,

\footnotetext{
*Correspondence: xywang@szu.edu.cn; bkkuai@fudan.edu.cn

${ }^{+}$Xiaoyan Wang and Xintong Xu contributed equally to this work.

${ }^{1}$ Guangdong Provincial Key Laboratory for Plant Epigenetics, College of Life Sciences and Oceanography, Shenzhen University, Shenzhen 518060, China ${ }^{2}$ State Key Laboratory of Genetic Engineering, School of Life Sciences, Fudan University, Shanghai 200438, China
}

FLOWERING CONTROL LOCUS A (FCA), FLOWERING LOCUS PA (FPA), FLOWERING LOCUS KH DOMAIN (FLK), FLOWERING LOCUS Y (FY), LUMINIDEPENDENS (LD), FLOWERING LOCUS VE (FVE), and FLOWERING LOCUS $D(F L D)$ repress FLC expression under both longday (LD) and short-day (SD) conditions [7, 8]. The MADSbox transcription factor FLC directly binds to the chromatin of floral integrator FLOWERING LOCUS T $(F T)$ and SUPPRESSOR OF OVEREXPRESSION OF CONSTANS 1 (SOC1) to repress flowering [9, 10]. FLC acts as a central floral repressor by converging the signal from different pathways in a dose-dependent manner [11]. Its expression is under sophisticated control at both transcriptional and posttranscriptional levels by diverse regulate factors [12-14]. Through massive studies, the $F L C$ locus not only shows the delicate regulation involving flowering control, but also provides an important platform for discovering epigenetic regulation of gene expression [14].

Members of the Teosinte Branched 1/Cycloidea/Proliferating Cell Factors (TCP) family are plant-specific transcription factors involved in many biological processes including 
flowering [15]. The Arabidopsis TCP family consists of 24 genes and can be further divided into two clades, class I and class II, based on their sequence features [16]. Involvement of the TCPs from both classes in regulating flowering has been reported in previous studies. For example, TCP15 promotes flowering by directly regulating $\mathrm{SOC} 1$ expression [17], and TCP4 functions as a transcriptional activator by directly binding to the CONSTANS $(\mathrm{CO})$ promoter to induce flowering $[18,19]$. Several TCPs can regulate floral transition by interacting with FT through protein-protein interaction [20, 21]. TCPs also affected flowering through circadian -related pathways. For example, TCP20 and TCP22 are positive regulators of CIRCADIAN CLOCK ASSOCIATED 1 (CCA1), while TCP21 represses CCA1 expression $[22,23]$. Despite the importance, the molecular mechanism underlying the TCP-FLC interaction remains to be elucidated.

Our previous work demonstrated TCP8, a member of class I TCPs, directly binds to the ISOCHORISMATE SYNTHASE 1 promoter to activate plant immune response [24]. Besides, TCP8 also binds and activates promoter of EF-TU RECEPTOR in planta during pathogenesis [25]. In addition, TCP8 interacts with NON-EXPRESSER of PR GENES 1 and SUPPRESSOR OF rps4-RLD1 via proteinprotein interaction after pathogen attack $[26,27]$. These results clearly showed a role of TCP8 participating in plantpathogen interaction. Other studies have also suggested TCP8 may have roles during plant development and yield determination [28-33], but its function in regulating flowering time is not clear. In this study, we investigate the role of TCP8 in flowering control by overexpression. We found that TCP8 overexpression delays plant flowering in an FLC-dependent manner. Furthermore, TCP8 and its homologs are indispensable for plant development.

\section{Results}

TCP8 is ubiquitously expressed during plant development To characterize the tissue-specific expression pattern of TCP8 at different developmental stages in detail, a $1.5 \mathrm{~kb}$ promoter region upstream of the start codon of TCP8 was fused with the GUS gene and the construct was transformed into Columbia-0 (Col-0). Several independent transgenic lines exhibited similar patterns of GUS expression. TCP8 showed a ubiquitous expression pattern in the transgenic plants-GUS signal was mainly detected in the vascular bundles in cotyledons, primary roots, hypocotyls and rosette leaves throughout development (Fig. 1a, d, e). Relatively higher TCP8 expression was detected in leaf primordia and stomatal guard cells (Fig. 1b, c), suggesting a potential role of TCP8 in tissue initiation and stomatal function. We then validated the histochemical GUS staining results by realtime quantitative PCR (RT-qPCR). Consistently, TCP8 expression was detected in all the tissues tested-including rosette leaves, cauline leaves, stem, inflorescence and root

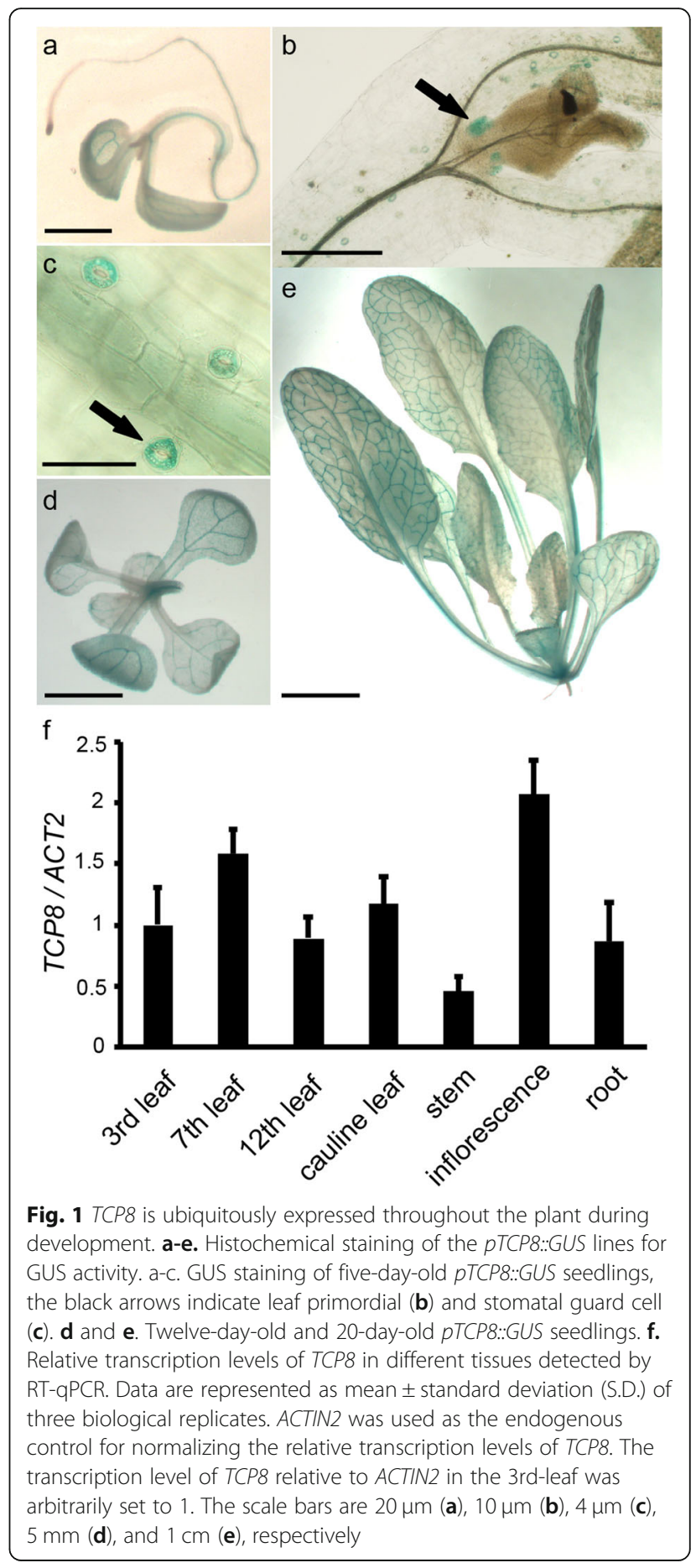

(Fig. 1f). Taken together, these results revealed the ubiquitous expression pattern of TCP8 throughout development and a potentially function in flowering.

\section{TCP8 overexpression delayed flowering under both LD and SD conditions}

To further investigate the role of TCP8, we overexpressed TCP8 using the constitutive $35 \mathrm{~S}$ cauliflower mosaic virus 
promoter (35S) in Col-0 and homozygous T3 progeny were analyzed. The 35S::TCP8 plants showed a clear lateflowering phenotype compared with wild-type (WT) Col-0 under the long-day (LD) condition, and the extent of delay well correlated with the relative transcription levels of TCP8 (Fig. 2a, b). A similar late-flowering phenotype was also observed for the 35S::TCP8 plants under the short-day (SD) condition (Fig. 2d). It is well accepted that lateflowering plants often generate more rosette leaves before flowering. Indeed, the TCP8 overexpression plants generated more rosette leaves than control plants both under LD and SD (Fig. 2c), demonstrating that TCP8 is a bona fide regulator of Arabidopsis flowering. Moreover, we observed retarded growth with the $35 S:: T C P 8$ individuals, although the final plant height of $35 S:: T C P 8$ were comparable to those of the WT (Additional file 1: Figure S1).
Next, we expressed TCP8 in Col-0 with the native promoter of TCP8 to eliminate the effect of ectopically expressed TCP8 on flowering. The $p T C P 8:: T C P 8$ plants phenocopied 35S::TCP8 plants by showing a delayed flowering compared with the WT control (Additional file 2: Figure S2), although the late-flowering phenotype was less obvious compared with the 35S:: TCP8 individuals, probably due to a lower expression levels of TCP 8 under its native promoter. Collectively, these data provide evidence for a role of TCP8 in regulating plant flowering.

\section{Overexpression of TCP8 up-regulates FLC mRNA level}

The delayed flowering phenotype of $35 \mathrm{~S}:: T C P 8$ plants under both LD and SD conditions suggest a putative role of TCP8 in the autonomous pathways. Thus, we

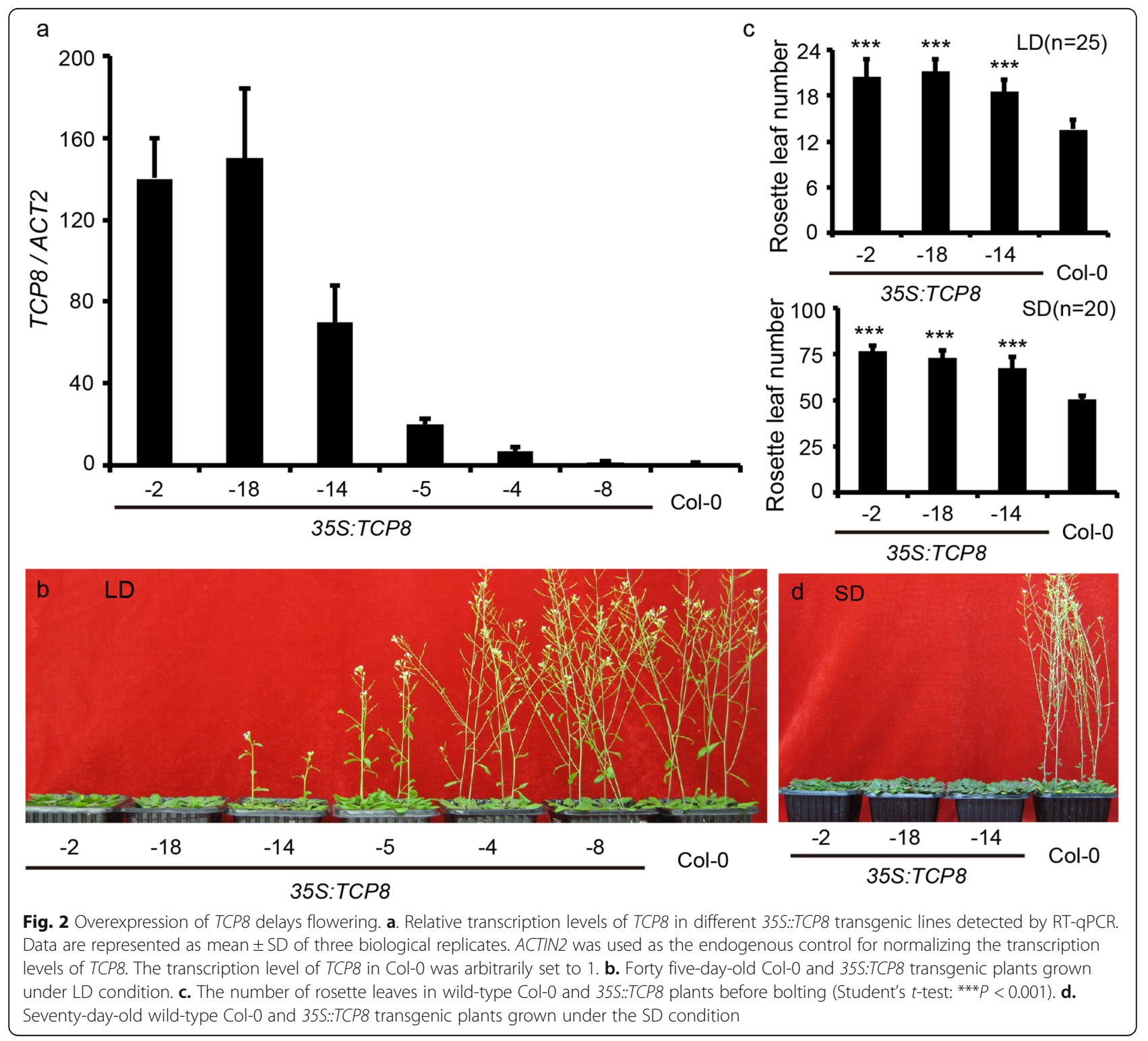


examined the transcription levels of $F L C$-a master repressor of flowering in the autonomous pathways-in the 35S::TCP8 lines. Consistent with our hypothesis, $F L C$ transcription level in the 35S::TCP8 lines increased by over two folds compared with WT control during early developmental stages (Fig. 3a). This result suggests that TCP8 may control flowering by upregulating FLC mRNA level.

Vernalization has been shown as an effective way to reduce native $F L C$ expression, so we checked whether vernalization could rescue the late-flowering phenotype of TCP8 overexpression plants. As expected, vernalization greatly rescued the delayed flowering of TCP8 overexpression plants (Fig. 3b). We observed no obvious difference in the number of roseate leaves between the 35S::TCP8 and control plants in the vernalization group (Fig. 3c), confirming a potential role of FLC in TCP8-mediated flowering control. It is of interest to note that the number of days after germination for flowering of TCP8 overexpression plants were still more than WT, presumably owing to the retarded growth caused by TCP8 overexpression (Fig. 3b). That is, vernalization specifically rescued the late-flowering phenotype caused by TCP8 overexpression, but had no effect on its growth hindering effect.

To further investigate the molecular mechanism of $T C P 8$ in controlling flowering, we tested the binding of TCP8 to FLC promoter in TCP8-GFP transgenic plants [24] using the chromatin immunoprecipitation (ChIP) assay and the in vitro electrophoretic mobility shift assay (EMSA) (Additional file 3: Figure S3). Neither the ChIP assay nor the EMSA detected an interaction between TCP8 and the $F L C$ promoter, suggesting that TCP8 indirectly regulated $F L C$ expression. Considering the Col-0 ecotype contains a fri-null allele, TCP8 may regulate genes upstream of FLC in the autonomous pathway, which were thought to cooperatively repress $F L C$ expression. Therefore, we measured the relative expression levels of $F C A$, $F P A, F L K, F Y, L D, F V E$, and $F L D$ in the TCP8 overexpression seedlings and observed moderate decreases in $F C A$, $F L K, L D$ and $F L D$ expression (Additional file 4: Figure S4a). In addition, a group of antisense long noncoding transcripts termed COOLAIR are reported to regulate FLC expression level [34]. But we did not detect a significant change of COOLAIR levels in TCP8 overexpression seedlings (Additional file 4: Figure S4b). Taken together, these analyses revealed that TCP8 overexpression indirectly upregulted FLC mRNA level, probably through the downregulation of a set of autonomous genes.

\section{FLC is required for TCP8-mediated flowering control}

To validate the role of FLC in TCP8-regulated flowering, we overexpressed TCP8 in an FLC loss-of-function mutant, $f l c$ 6. TCP8 overexpression had no obvious effect on the flowering of $f l c-6-$ no significant difference in rosette leaves number was observed between the $f l c-6$ and $35 S:: T C P 8 / f l c-6$ individuals-despite high expression of TCP8 was detected in 35S::TCP8/flc-6 plants (Fig. 4a-c). We then crossed the
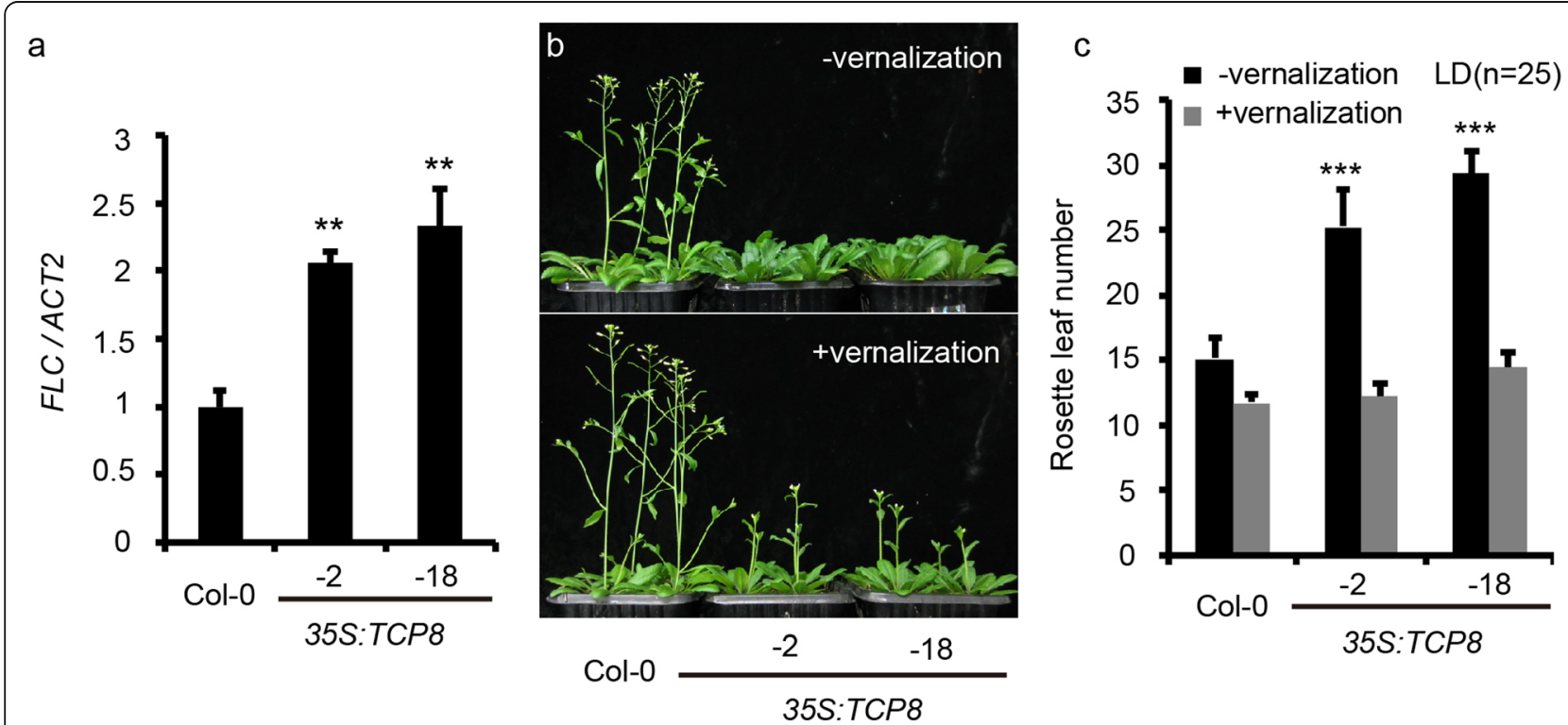

Fig. 3 Overexpression of TCP8 up-regulates FLC mRNA level. a. Relative transcription levels of FLC in 35S::TCP8 seedlings detected by RT-qPCR. Data are shown as mean \pm SD of three biological replicates. ACTIN2 was used as the endogenous control for normalizing the transcription levels of FLC. The transcription level of FLC in wild-type Col-0 was arbitrarily set to 1. (Student's $t$ test: ${ }^{* *} P<0.01$ ). b. Forty-day-old wild-type Col-0 and 35 S::TCP8 plants grown under LD condition with or without vernalization. c. The number of rosette leaves in wild-type Col-0 and 35 S::TCP8 plants before bolting. (Student's t-test, ${ }^{* *} P<0.01,{ }^{* * *} P<0.001$ ) 


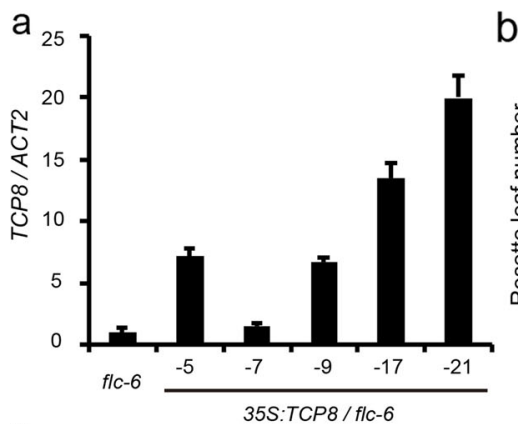

C

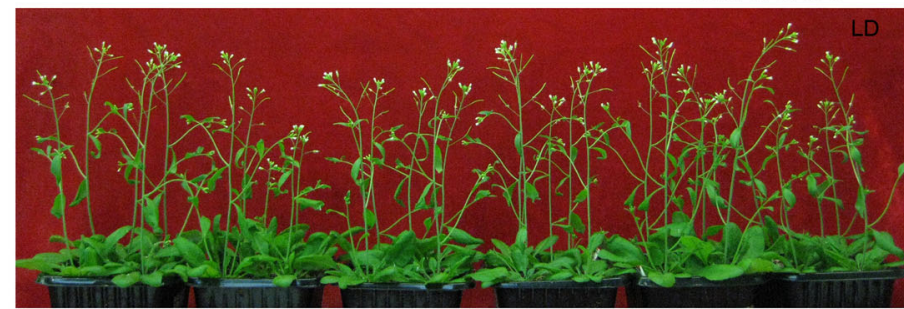

flc- 6

$\begin{array}{lll}-7 & -9 & -17\end{array}$

35S:TCP $8 / f 1 c-6$

d

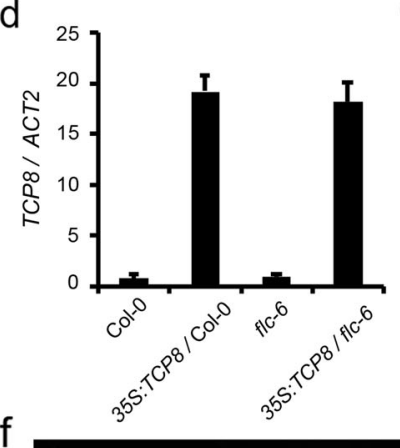

e

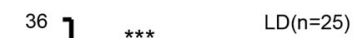

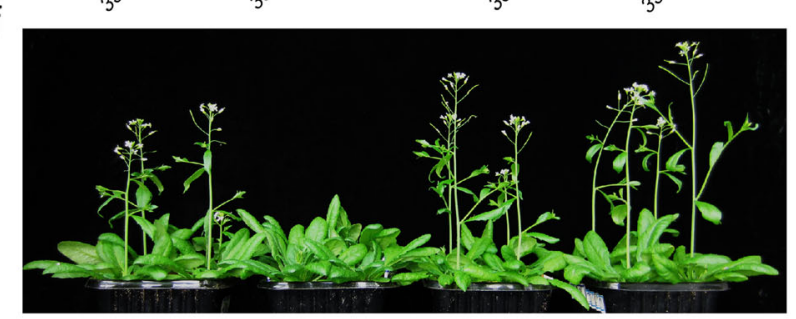

Col-0 35S:TCP8 / Col-0

flc- 6

35S:TCP8/flc-6

Fig. 4 FLC is required for the TCP8 function in regulating flowering time. a. Relative transcription levels of TCP8 in flc-6 and 355 ::: TCP8/flc-6 detected by RT-qPCR. Data are represented as mean \pm S.D. of three biological replicates. ACTIN2 was used as the endogenous control for normalizing the transcription levels of TCP8. The transcription level of TCP8 in flc- 6 was arbitrarily set to 1 . b. The number of rosette leaves in flc- 6 and 35S::TCP8/flc-6 plants before bolting. c. Forty-day-old flc-6 and 35S::TCP8/flc-6 plants grown under LD condition. d. Transcription levels of TCP8 in wild-type Col-0, 355::TCP8, flC-6 and 355:::TCP8/flC-6 individuals. ACTIN2 was used as the endogenous control for data normalization and the transcription level of TCP8 is represented as mean \pm S.D. of three biological replicates. The transcription level of TCP8 in Col-0 was arbitrarily set to 1. e. The number of rosette leaves in wild-type Col-0, 35S::TCP8, flc-6 and 35S::TCP8/flc-6 before bolting. (Student's t-test, $* * * P<0.001$, ns $=$ not significant). f. Forty-day-old wild-type Col-0, 355::TCP8, flc-6 and 355::TCP8/flc-6 plants grown under LD condition

35S::TCP8 transformants in Col-0 with $f l c-6$. In the F2 segregation population, TCP8 overexpression in flc-6 background showed comparative flowering time with $f l c-6$, while TCP8 overexpression in FLC WT background showed significant delayed flowering (Fig. 4d-f), suggesting that a functional $F L C$ gene is required for TCP8 function. We obtained similar results in $p T C P 8: T C P 8$ and $f l c-6$ crossed F2 progeny
(Additional file 5: Figure S5). Therefore, FLC is essential for the TCP8-mediated flowering control.

\section{Dominant repression by $T C P 8$ leads to various growth} defects

A TCP8 mutant generated by T-DNA insertion (tcp8-1, CS875709) was obtained from ABRC to investigate the 
role of TCP8 in flowering. However, we observed no visible defect in flowering and development (Additional file 6: Figure S6), which may be owing to the functional redundancy among TCP family members, as proposed in previous studies [35]. To overcome this redundancy, we fused the EAR motif to the C-terminus of TCP8 and overexpressed the TCP8-EAR fusion protein in Col-0 to mediate dominant repression by TCP8 (Fig. 5a). The 35S::TCP8-EAR plants exhibited severe developmental defects-the growth of most plants was arrested at the seedling stage (Fig. 5c). The survived 35S: TCP8-EAR seedlings failed to develop normal leaves (Fig. 5d). Similarly, we observed a moderate level of growth defect with the pTCP8::TCP8-EAR seedlings, which had dark green leaves, hyponastic cotyledons, and shorter primary roots (Fig. 5e, f); some pTCP8::TCP8$E A R$ seedlings could develop curved true leaves but few made to the reproductive stage (Fig. 5h). The adult plants of $p T C P 8:: T C P 8-E A R$ were considerably smaller than Col-0 and could not develop a normal inflorescence-the three outer whorls of the $p T C P 8:: T C P 8-E A R$ flower were fused and the irregular gynoecia was exposed (Fig. 5i, j). The pTCP8::TCP8-EAR plants also failed to develop viable seeds. Taken together, these results point to an indispensable role of TCP8 in maintaining normal plant growth and flower development.

\section{Discussion}

Functional redundancy often hinders the analysis of the TCP family members [15]. For example, TCP8, TCP14, and TCP15 are functionally redundant in regulating development and immune response [25-28, 30], however, TCP8 and TCP15 may play different roles in controlling flowering. Overexpression of TCP15 promoted early flowering and the tcp 15 loss-of-function mutant showed delayed flowering [17]. In the present study, the tcp 8 single mutant had no obvious phenotype but TCP8 overexpression led to delayed flowering (Fig. 2b-d). According

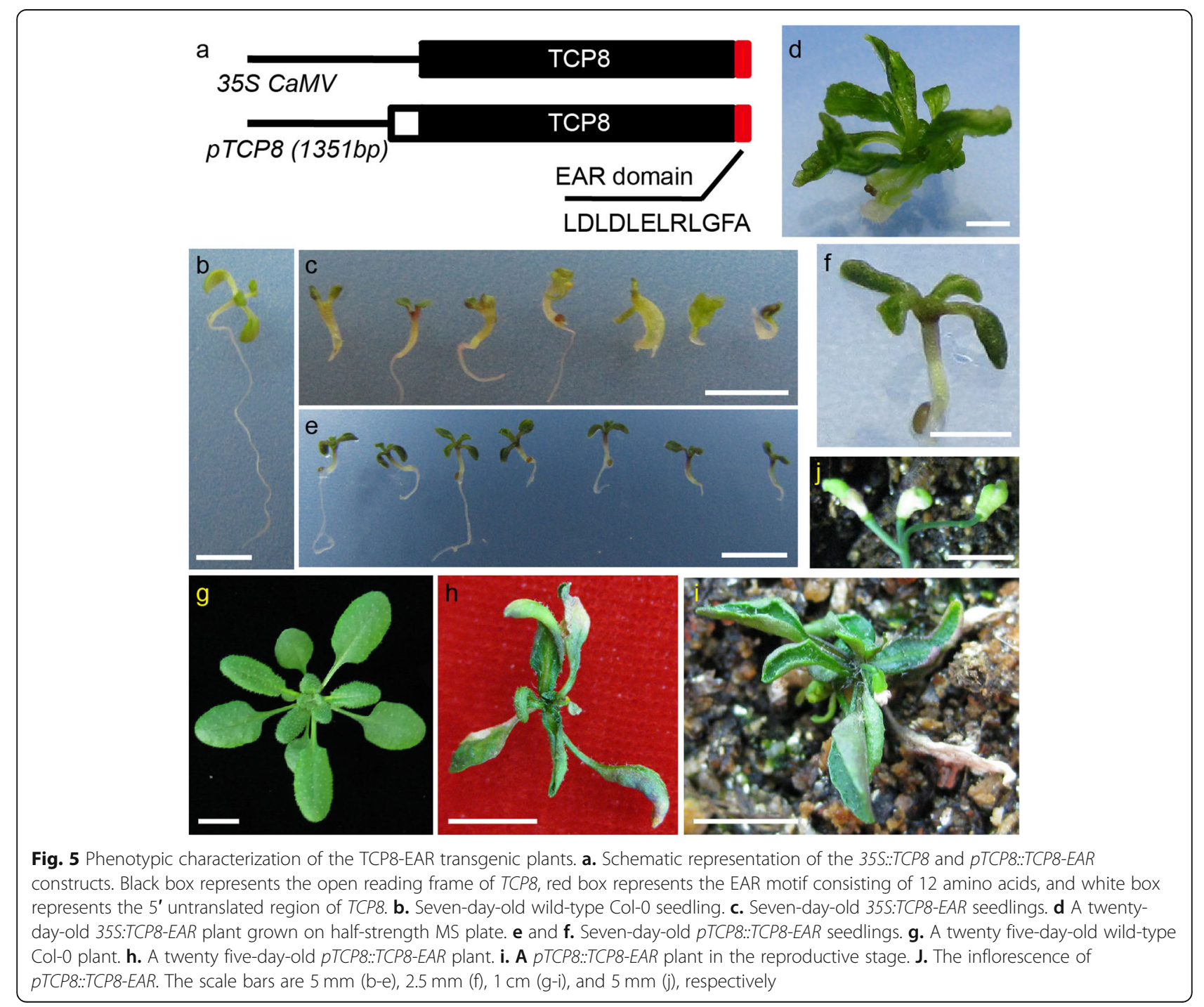


to previous studies, TCP15 directly activates $\mathrm{SOC1}$ expression [17], and TCP8 functions upstream of FLC (Fig. 4), suggesting that TCP8 functions upstream of SOC1. In contrast to that observed with the immune response, TCP8 and TCP15 may have no redundancy in flowering regulation. TCP23 overexpression could also delay flowering under LD condition [36], suggesting that TCP23 and TCP8 may have overlapping functions in flowering control. To overcome the functional redundancy among TCP members, the chimeric TCP8-EAR protein was expressed in WT Col-0 to introduce dominant TCP8 repression, which resulted in various growth defects in the transgenic plants (Fig. 5). Compared with TCP14 and TCP15 dominant repression [37-39], the TCP8-EAR plants exhibited more severe developmental defects. This observation is consistent with the ubiquitous expression pattern of TCP8 during seedling development (Fig. 1).

TCP8 overexpression delayed flowering under both LD and SD conditions and TCP8 functions upstream of FLC, suggesting that TCP8 may play a role in the autonomous pathways. Consistent with this hypothesis, we found that the expression levels of several known autonomous pathway regulators, including $F C A, F L K, L D$, and $F L D$, were downregulated in the TCP8 overexpression lines (Additional file 4: Figure S4a), suggesting that TCP8 may indirectly affect FLC expression through regulating these genes. Consistent with this assumption, we failed to detect a direct interaction between TCP8 and the promoter of $F L C$ in the EMSA and ChIP assay. Previous studies have suggested that the autonomous pathway factors mainly regulate $F L C$ expression via post-transcriptional RNAprocessing and epigenetic mechanisms [40-42]. Future work is required to characterize the interaction between TCP 8 and FLC in detail. TCP14 and TCP15 have been shown to interact with MODIFIER OF snc1-1 (MOS1), which directly interacts with Suppressor of FRIGIDA 4-a transcriptional activator of $F L C$ [28, 43]. In this scenario, TCP8 may also regulate $F L C$ mRNA level through interaction with MOS1. Alternatively, TCP8 may compete the interaction between TCP14, TCP15 with MOS1, as protein-protein interactions between TCPs are prevalent $[24,35]$. Previous studies have shown that the AP2domain transcription factors TEMPRANILLO1 (TEM1) and TEM2 inhibit flowering under LD condition through direct transcriptional repression of $F T$ [44]. Moreover, TEMs also regulate flowering involving the gibberellin levels and miR172, both required to orchestrate floral transition under SD conditions $[45,46]$. Interestingly, we detected up-regulated levels of TEM1 and TEM2 in TCP8 overexpression lines under LD condition (Additional file 7: Figure S7). Examining the putative relationship between TCP8 and TEMs may provide new clues for their functions.
In this study, we found that TCP8 overexpression inhibited plant growth in a dose-dependent manner (Additional file 1: Figure S1a). The 35S::TCP8 plants showed retarded growth earlier in development but the adult plants established a normal plant height comparable to the wild-type control (Additional file 1: Figure S1b). TCP8 and other TCP genes are known to participate in cell-cycle control, presumably through regulating CYCA1;1 and CYCA2;3 [28, 33, 47]. Thus, the growth inhibition in $355:: T C P 8$ may be related to the misregulation of cell-cycle. Vernalization could reverse the lateflowering phenotype but not the growth inhibition of $35 S:: T C P 8$ plants, suggesting independent regulation of flowering time and cell-cycle by TCP8, and TCP8related cell-cycle regulation has negligible role in flowering control in our study.

\section{Conclusions}

Our study demonstrates that TCP8 regulates plant flowering in an FLC-dependent manner. The findings of this study expand our knowledge on the molecular basis of how $T C P s$, especially $T C P 8$, control flowering time and provide evidence for the interaction between TCP8 and FLC.

\section{Methods}

\section{Plant materials and growth conditions}

All mutants and transgenic lines used in this study were in the Arabidopsis thaliana ecotype Col-0 background. The T-DNA insertion mutant tcp8-1 (CS875709) was obtained from the Arabidopsis Biological Resource Center (ABRC, https://abrc.osu.edu/) and genotyped as previously reported [24]. For transgenic plants, the corresponding plasmid was transformed into WT Col-0 or flc-6 (SALK 041126) by floral dipping and the transgenic plants were selected by half-strength Murashige and Skoog (MS) medium supplemented with proper antibiotic. For TCP8 overexpression lines, homozygous T3 transgenic plants were used for further analysis. For the co-segregation analysis, 35S::TCP8 or $p T C P 8:: T C P 8$ plants crossed with $f l c-6$, and the resulting F2 progenies in each population were genotyped. All primers used for genotyping are listed in Additional file 8: Table S1.

Imbibed seeds were sowed and stratified in a cold room at $4{ }^{\circ} \mathrm{C}$ for 3 days to break dormancy and then transferred to $22 \pm 1{ }^{\circ} \mathrm{C}$ at a light intensity of approximately $120 \mu \mathrm{mol} \mathrm{m}^{-2} \mathrm{~s}^{-1}$, with a $16 \mathrm{~h}$ light $/ 8 \mathrm{~h}$ dark photoperiod for LD and $8 \mathrm{~h}$ light/16 $\mathrm{h}$ dark photoperiod for SD. The number of rosette leaves was counted after the main stem has bolted to $2 \mathrm{~cm}$. For the vernalization treatment, the imbibed seeds were grown on halfstrength MS medium at $4{ }^{\circ} \mathrm{C}$ for 4 weeks under dim light and then transferred to $22 \pm 1{ }^{\circ} \mathrm{C}$ and grown under $\mathrm{LD}$ condition. 


\section{Vector construction}

A $1582 \mathrm{bp}$ promoter fragment upstream of the TCP8 translation start site was amplified by PCR using primers pTCP8-1582-S/A, and then cloned into the pCAMBIA1301 (GeneBank accession number AF234297) vector through KpnI/BglII double digestion to generate the pTCP8::GUS construct (the TCP8 promoter-driven GUS expression construct). For TCP8 overexpression, the coding sequence (CDS) of TCP8 was amplified using primers OE-TCP8-S/A and cloned into the $\mathrm{pCHF} 3$ vector by $S a c \mathrm{I} / \mathrm{BamHI}$ double digestion to generate the 35S::TCP8 vector. For $p$ TCP8::TCP8 vector construction, a DNA fragment containing the TCP8 coding region as well as the $1582 \mathrm{bp}$ promoter region was amplified from Col-0 genomic DNA using primers pTCP8-TCP8-S/A and cloned into the pCAMBIA1301 through PstI/BstpI double digestion. To construct the 35S::TCP8-EAR vector, DNA fragment containing the EAR motif was synthesized using primers EAR-S/A and cloned into the $35 S:: T C P 8$ vector via BamHI/Pst double digestion. To construct the $p T C P 8:: T C P 8-E A R$ vector, a $1351 \mathrm{bp}$ promoter fragment upstream of the TCP8 translation start site was amplified using primers pTCP8-1351-S/A and cloned into the 35S::TCP8-EAR vector through EcoRI/ $\mathrm{S} a \mathrm{I}$ double digestion. Primers used for vector construction are summarized in Additional file 8: Table S1.

\section{Histochemical GUS assays}

For the histochemical detection of GUS activity, plant tissues were immersed in the 5-Bromo-4-chloro-3-indolyl $\beta$-D-glucuronic acid (X-Gluc) solution (containing $750 \mathrm{mg} \mathrm{ml}^{-1} \quad \mathrm{X}$-Gluc, $\quad 0.2 \mathrm{mM} \quad \mathrm{K}_{3} \mathrm{Fe}(\mathrm{CN})_{6}, \quad 0.2 \mathrm{mM}$ $\mathrm{K}_{4} \mathrm{Fe}(\mathrm{CN})_{6}$ and $0.2 \%$ Triton $\left.\mathrm{X}-100, \mathrm{pH}=7.2\right)$ in the vacuum for $15 \mathrm{~min}$ at room temperature and incubated at $37^{\circ} \mathrm{C}$ overnight. The samples were then washed with $70 \%$ ethanol several times until transparent before examined under the microscope.

\section{RNA extraction and quantitative real-time PCR}

To investigate the transcript profile of TCP8, different tissues of 40-day-old WT Col-0 plants grown under LD condition were harvested. For detecting the transcript levels of TCP8 in Col-0 and the 35S::TCP8, rosette leaves were collected. To detect the transcript levels of FLC and genes involved in the autonomous pathways, tenday-old seedlings grown under LD condition were collected. Total RNA was extracted from the tissues of Col- 0 and the transgenic lines using the TRIzol reagent (Invitrogen), DNase I (TaKaRa) was used to wipe out genomic DNA. Two micrograms of total RNA of each sample was used for first-strand cDNA synthesis with M-MLV reverse transcriptase (TaKaRa).

RT-qPCR was performed as previously described [48]. Briefly, reverse-transcribed cDNA was used as the template for RT-qPCR. The RT-qPCR reactions were performed using SYBR Green (TaKaRa) on an iCycler (Bio-Rad) following the manufacturer's instructions. ACTIN2 was used as the endogenous control for normalizing the transcript levels of the tested genes. All experiments were performed in three independent biological replicates and three technical replicates. Data are represented as mean \pm standard deviation (S.D.). Asterisks indicate significant differences relative to control $\left({ }^{*} P<0.05,{ }^{* *} P<0.01,{ }^{* * *} P<0.001\right.$, Student's $t$-test). Primers used for RT-qPCR are listed in Additional file 8: Table S1.

\section{Supplementary information}

Supplementary information accompanies this paper at https://doi.org/10. 1186/s12870-019-2157-4.

Additional file 1: Figure S1. TCP8 overexpression hinders plant growth a. Twenty-day-old wild-type Col-0 and 35S:::TCP8 transgenic plants grown in LD condition. b. Sixty-day-old wild-type Col-0 and 35S::TCP8 transgenic plants grown in LD condition. (LD: $16 \mathrm{~h}$ of light / $8 \mathrm{~h}$ of dark).

Additional file 2: Figure S2. $P$ TCP8::TCP8 delays flowering. a. Relative transcription levels of TCP8 in different PTCP8:: TCP8 transgenic lines detected by RT-qPCR. Data are represented as mean \pm SD of three biological replicates. ACTIN2 was used as the endogenous control for normalizing the transcription levels of TCP8. The transcription level of TCP8 in Col-0 was arbitrarily set to 1 . b. Forty-day-old wild-type Col-0 and pTCP8::TCP8 transgenic plants grown in LD condition. c. The number of rosette leaves in wild-type Col-0 and pTCP8::TCP8 plants before bolting (Student's $t$-test: ${ }^{*} P<0.05$, ${ }^{* *} P<0.01$, ${ }^{* * *} P<0.001$ ).

Additional file 3: Figure S3. TCP8 failed to bind the FLC promoter in vitro and in vivo. a. EMSA detection of TCP8 binding to FLC promoter fragment. The ICS1 promoter containing a TCP binding site was used as a positive control. b. TCP8 failed to co-precipitated DNA fragments around FLC locus in the chromatin immunoprecipitation (ChIP) assay. The ICSI promoter was used as a positive control. The $18 \mathrm{~S}$ rRNA gene was used to normalize the quantitative $P C R$ results for each of the ChIP samples. Values are means \pm SD of three quantitative PCR measurements.

Additional file 4: Figure S4. Detection of autonomous pathway genes and COOLAIR levels in TCP8 overexpression plants. a. Overexpression of TCP8 down-regulates a set of autonomous pathway genes expression. Relative transcription levels of autonomous pathway genes in TCP8 overexpression transgenic lines detected by RT-qPCR. Data are represented as mean \pm SD of three biological replicates. ACTIN2 was used as the endogenous control for normalizing the transcription levels of genes detected. The transcription level of each gene in Col-0 was arbitrarily set to 1. (Student's $t$ test: $\left.{ }^{*} P<0.05\right)$ b. Relative transcription levels of different COOLAIR isoforms in TCP8 overexpression transgenic lines detected by RT-qPCR. Data are represented as mean \pm SD of three biological replicates. ACTIN2 was used as the endogenous control for normalizing the transcription levels of different COOLAIR isoforms. The transcription level of COOLAIR in Col-0 was arbitrarily set to 1.

Additional file 5: Figure S5. The flowering phenotypes of $p$ TCP8:::TCP8 and flc-6 crossed F2 progenies.

Additional file 6: Figure S6. The flowering phenotypes of tcp8-1 in LD condition. a. Forty-day-old plants of different genotypes grown in LD condition. b. The number of rosette leaves in Col-0 and tcp8-1 plants before bolting.

Additional file 7: Figure S7. Detection of TEM1 and TEM2 expression levels in TCP8 overexpression plants in LD condition. Forty-day-old plants of different genotypes grown in LD condition. Relative transcription levels of TEM1 and TEM2 in TCP8 overexpression transgenic lines detected by RT-qPCR. Data are represented as mean \pm SD of three biological replicates. ACTIN2 was used as the endogenous control for normalizing the 
transcription levels of genes detected. The transcription level of each gene in Col-0 was arbitrarily set to 1. (Student's $t$ test: ${ }^{*} P<0.05$ ).

Additional file 8: Table S1. Primers used in this paper.

\section{Abbreviations}

35S CaMV: 35 S cauliflower mosaic virus: CCA1: CIRCADIAN CLOCK ASSOCIATED 1; ChIP: Chromatin immunoprecipitation; CO: CONSTANS; Col0: Columbia-0; EMSA: Electrophoretic mobility shift assay; FCA: FLOWERING CONTROL LOCUS A; FLC: FLOWERING LOCUS C; FLD: FLOWERING LOCUS D; FLK: FLOWERING LOCUS KH DOMAIN; FPA: FLOWERING LOCUS PA; FRI: FRIGIDA; FT: FLOWERING LOCUS T; FVE: FLOWERING LOCUS VE; FY: FLOWERING LOCUS Y; GFP: Green fluorescent protein; GUS: $\beta$ glucuronidase; LD: LUMINIDEPENDENS; RT-gPCR: Real-time quantitative polymerase chain reaction; SOC1: SUPPRESSOR OF OVEREXPRESSION OF CONSTANS 1; TCP: Teosinte Branched 1 / Cycloidea / Proliferating Cell Factors; TEM1/2: TEMPRANILLO 1/2

\section{Acknowledgements}

We thank Dr. Dabing Zhang for sharing the seeds of flc-6.

\section{Authors' contributions}

XYW, BXM and BKK designed the study. XYW, XTX, XWM, LYZ and JCZ performed the experiments. XYW, XTX and BKK analyzed the data. BXM provided critical reagents and equipment for the study. XYW and BKK wrote the manuscript. All authors read and approved the final manuscript.

\section{Funding}

This work was supported by Natural Science Foundation of Guangdong Province (2018A030313966) and Shenzhen Science and Technology Innovation Committee (JCYJ20170818100038326). The funders had no role in the design of the study and collection, analysis, and interpretation of data and in writing the manuscript.

\section{Availability of data and materials}

The datasets generated and analyzed during the current study are available from the corresponding author ( $X$. Wang) on reasonable request.

\section{Ethics approval and consent to participate}

Not applicable.

\section{Consent for publication}

Not applicable.

\section{Competing interests}

The authors declare that they have no competing interests.

\section{Received: 2 July 2019 Accepted: 21 November 2019}

\section{Published online: 03 December 2019}

\section{References}

1. Simpson GG, Dean C. Flowering - Arabidopsis, the rosetta stone of flowering time? Science. 2002;296(5566):285-9.

2. Michaels SD. Flowering time regulation produces much fruit. Curr Opin Plant Biol. 2009;12(1):75-80

3. Srikanth A, Schmid M. Regulation of flowering time: all roads lead to Rome. Cell Mol Life Sci. 2011;68(12):2013-37.

4. Kazan K, Lyons R. The link between flowering time and stress tolerance. J Exp Bot. 2016;67(1):47-60.

5. Michaels SD, Amasino RM. FLOWERING LOCUS C encodes a novel MADS domain protein that acts as a repressor of flowering. Plant Cell. 1999;11(5): 949-56.

6. Kim DH, Doyle MR, Sung S, Amasino RM. Vernalization: winter and the timing of flowering in plants. Annu Rev Cell Dev Bi. 2009;25:277-99.

7. Simpson GG. The autonomous pathway: epigenetic and post-transcriptional gene regulation in the control of Arabidopsis flowering time. Curr Opin Plant Biol. 2004:7(5):570-4

8. Cheng JZ, Zhou YP, LV TX, Xie CP, Tian CE. Research progress on the autonomous flowering time pathway in Arabidopsis. Physiol Mol Biol Plants. 2017;23(3):477-85
9. Helliwell CA, Wood CC, Robertson M, James Peacock W, Dennis ES. The Arabidopsis FLC protein interacts directly in vivo with SOC1 and FT chromatin and is part of a high-molecular-weight protein complex. Plant J. 2006:46(2):183-92.

10. Searle I, He Y, Turck F, Vincent C, Fornara F, Krober S, Amasino RA, Coupland $G$. The transcription factor FLC confers a flowering response to vernalization by repressing meristem competence and systemic signaling in Arabidopsis. Genes Dev. 2006;20(7):898-912.

11. Hepworth J, Dean C. Flowering locus C's lessons: conserved chromatin switches underpinning developmental timing and adaptation. Plant Physiol. 2015:168(4):1237-45.

12. Amasino R. Seasonal and developmental timing of flowering. Plant J. 2010; 61(6):1001-13.

13. He Y. Chromatin regulation of flowering. Trends Plant Sci. 2012;17(9):556-62

14. Whittaker C, Dean C. The FLC Locus: A Platform for Discoveries in Epigenetics and Adaptation. Annu Rev Cell Dev Biol. 2017;33:555-75.

15. Nicolas M, Cubas P. TCP factors: new kids on the signaling block. Curr Opin Plant Biol. 2016;33:33-41

16. Martin-Trillo M, Cubas P. TCP genes: a family snapshot ten years later. Trends Plant Sci. 2010;15(1):31-9.

17. Lucero LE, Manavella PA, Gras DE, Ariel FD, Gonzalez DH. Class I and class II TCP transcription factors modulate SOC1-dependent flowering at multiple levels. Mol Plant. 2017;10(12):1571-4.

18. Liu J, Cheng X, Liu P, Li D, Chen T, Gu X, Sun J. MicroRNA319-regulated TCPs interact with FBHs and PFT1 to activate CO transcription and control flowering time in Arabidopsis. PLoS Genet. 2017;13(5):e1006833.

19. Kubota A, Ito S, Shim JS, Johnson RS, Song YH, Breton G, Goralogia GS, Kwon MS, Laboy Cintron D, Koyama T, et al. TCP4-dependent induction of CONSTANS transcription requires GIGANTEA in photoperiodic flowering in Arabidopsis. PLoS Genet. 2017;13(6):e1006856.

20. Niwa M, Daimon Y, Kurotani Kl, Higo A, Pruneda-Paz JL, Breton G, Mitsuda $N$, Kay SA, Ohme-Takagi $M$, Endo $M$, et al. BRANCHED1 interacts with FLOWERING LOCUS T to repress the floral transition of the axillary meristems in Arabidopsis. Plant Cell. 2013;25(4):1228-42.

21. Ho WWH, Weigel D. Structural features determining flower-promoting activity of Arabidopsis FLOWERING LOCUS T. Plant Cell. 2014;26(2):552-64.

22. Wu JF. Tsai HL, Joanito I, Wu YC, Chang CW, Li YH, Wang Y, Hong JC, Chu JW, Hsu CP et al: LWD-TCP complex activates the morning gene CCA1 in Arabidopsis. Nat Commun. 2016;7:13181.

23. Pruneda-Paz JL, Breton G, Para A, Kay SA. A functional genomics approach reveals CHE as a component of the Arabidopsis circadian Clock. Science. 2009;323(5920):1481-5.

24. Wang X, Gao J, Zhu Z, Dong X, Wang X, Ren G, Zhou X, Kuai B. TCP transcription factors are critical for the coordinated regulation of isochorismate synthase 1 expression in Arabidopsis thaliana. Plant J. 2015; 82(1):151-62.

25. Spears BJ, Howton TC, Gao F, Garner CM, Mukhtar MS, Gassmann W. Direct regulation of the EFR-dependent immune response by Arabidopsis TCP transcription factors. Mol Plant-Microbe Interact. 2019:32(2):540-9.

26. Kim SH, Son GH, Bhattacharjee S, Kim HJ, Nam JC, Nguyen PD, Hong JC, Gassmann W. The Arabidopsis immune adaptor SRFR1 interacts with TCP transcription factors that redundantly contribute to effector-triggered immunity. Plant J. 2014:78(6):978-89.

27. Li M, Chen H, Chen J, Chang M, Palmer IA, Gassmann W, Liu F, Fu ZQ. TCP transcription factors interact with NPR1 and contribute redundantly to systemic acquired resistance. Front Plant Sci. 2018;9:1153.

28. Zhang N, Wang Z, Bao Z, Yang L, Wu D, Shu X, Hua J. MOS1 functions closely with TCP transcription factors to modulate immunity and cell cycle in Arabidopsis. Plant J. 2018;93(1):66-78.

29. Zhang G, Zhao H, Zhang C, Li X, Lyu Y, Qi D, Cui Y, Hu L, Wang Z, Liang Z, et al. TCP7 functions redundantly with several class I TCPs and regulates endoreplication in Arabidopsis. J Integr Plant Biol. 2019;61(11):1151-70.

30. Daviere JM, Wild M, Regnault T, Baumberger N, Eisler H, Genschik P, Achard P. Class I TCP-DELLA interactions in inflorescence shoot apex determine plant height. Curr Biol. 2014;24(16):1923-8.

31. van Es SW, van der Auweraert EB, Silveira SR, Angenent GC, van Dijk AD , Immink RGH. Comprehensive phenotyping reveals interactions and functions of Arabidopsis thaliana TCP genes in yield determination. Plant J. 2019;99(2):316-28.

32. Danisman $S$, van der Wal F, Dhondt S, Waites $R$, de Folter $S$, Bimbo A, van Dijk AD, Muino JM, Cutri L, Dornelas MC, et al. Arabidopsis class I and class 
II TCP transcription factors regulate jasmonic acid metabolism and leaf development antagonistically. Plant Physiol. 2012;159(4):1511-23.

33. Aguilar-Martinez JA, Sinha N. Analysis of the role of Arabidopsis class I TCP genes AtTCP7, AtTCP8, AtTCP22, and AtTCP23 in leaf development. Front Plant Sci. 2013;4:406.

34. Wu Z, letswaart R, Liu F, Yang H, Howard M, Dean C. Quantitative regulation of FLC via coordinated transcriptional initiation and elongation. Proc Natl Acad Sci U S A. 2016;113(1):218-23.

35. Danisman $S$, van Dijk AD, Bimbo A, van der Wal F, Hennig $L$, de Folter $S$, Angenent GC, Immink RG. Analysis of functional redundancies within the Arabidopsis TCP transcription factor family. J Exp Bot. 2013;64(18):5673-85.

36. Balsemao-Pires E, Andrade LR, Sachetto-Martins G. Functional study of TCP23 in Arabidopsis thaliana during plant development. Plant Physiol Biochem. 2013;67C:120-5.

37. Li ZY, Li B, Dong AW. The Arabidopsis transcription factor AtTCP15 regulates endoreduplication by modulating expression of key cell-cycle genes. Mol Plant. 2012;5(1):270-80.

38. Uberti-Manassero NG, Lucero LE, Viola IL, Vegetti AC, Gonzalez DH. The class I protein AtTCP15 modulates plant development through a pathway that overlaps with the one affected by CIN-like TCP proteins. J Exp Bot. 2012; 63(2):809-23.

39. Kieffer M, Master V, Waites R, Davies B. TCP14 and TCP15 affect internode length and leaf shape in Arabidopsis. Plant J. 2011;68(1):147-58.

40. Baurle I, Dean C. Differential interactions of the autonomous pathway RRM proteins and chromatin regulators in the silencing of Arabidopsis targets. S. 2008;3(7):e2733.

41. Baurle I, Smith L, Baulcombe DC, Dean C. Widespread role for the floweringtime regulators FCA and FPA in RNA-mediated chromatin silencing. Science. 2007;318(5847):109-12.

42. Tian YK, Zheng $H$, Zhang F, Wang SL, Ji XR, Xu C, He YH, Ding Y. PRC2 recruitment and $\mathrm{H} 3 \mathrm{~K} 27 \mathrm{me} 3$ deposition at FLC require FCA binding of COOLAIR. Sci Adv. 2019;5(4):eaau7246.

43. Bao Z, Zhang N, Hua J. Endopolyploidization and flowering time are antagonistically regulated by checkpoint component MAD1 and immunity modulator MOS1. Nat Commun. 2014:5:5628.

44. Castillejo C, Pelaz S. The balance between CONSTANS and TEMPRANILLO activities determines FT expression to trigger flowering. Curr Biol. 2008; 18(17):1338-43.

45. Aguilar-Jaramillo AE, Marin-Gonzalez E, Matias-Hernandez L, Osnato M, Pelaz S, Suarez-Lopez P. TEMPRANILLO is a direct repressor of the microRNA miR172. Plant J. 2019;100(3):522-35.

46. Osnato M, Castillejo C, Matias-Hernandez L, Pelaz S. TEMPRANILLO genes link photoperiod and gibberellin pathways to control flowering in Arabidopsis. Nat Commun. 2012;3:808.

47. Zhang T, Qu Y, Wang H, Wang J, Song A, Hu Y, Chen S, Jiang J, Chen F. The heterologous expression of a chrysanthemum TCP-P transcription factor CmTCP14 suppresses organ size and delays senescence in Arabidopsis thaliana. Plant Physiol Biochem. 2017;115:239-48.

48. Yu J, Wang XY, Wei QA, Kuai BK. Identification of regulatory cis-elements upstream of AtNPR1 that are responsive to Probenazole treatment in transgenic tobacco plants. J Plant Biol. 2010;53(4):282-90.

\section{Publisher's Note}

Springer Nature remains neutral with regard to jurisdictional claims in published maps and institutional affiliations.

\section{Ready to submit your research? Choose BMC and benefit from}

- fast, convenient online submission

- thorough peer review by experienced researchers in your field

- rapid publication on acceptance

- support for research data, including large and complex data types

- gold Open Access which fosters wider collaboration and increased citations

- maximum visibility for your research: over $100 \mathrm{M}$ website views per year

At BMC, research is always in progress.

Learn more biomedcentral.com/submissions 